\title{
Etiología de la cirrosis: los cambios epidemiológicos entre los períodos 1995-2002 y 2010-2017
}

\author{
Daniel S García, ${ }^{1}$ Yamila Martínez Artola, ${ }^{1}$ Daniel A Poncino, ${ }^{1}$ María Ferreira Rubino, ${ }^{2}$ \\ Rafael Escobar, ${ }^{3}$ Marina Khoury ${ }^{4}$ \\ ${ }^{1}$ Servicio de Hepatología, Sanatorio Dr. Julio Méndez. OBSBA. \\ ${ }^{2}$ Servicio de Ecografía, Sanatorio Dr. Julio Méndez. OBSBA. \\ ${ }^{3}$ Servicio de Gastroenterología y Endoscopía Digestiva, Sanatorio Dr. Julio Méndez. OBSBA. \\ ${ }^{4}$ Estadistica y Metodología de la Investigación, Instituto de Investigaciones Médicas Alfredo Lanari, Facultad de Medicina, Universidad de Buenos Aires. \\ Ciudad Autónoma de Buenos Aires, Argentina.
}

Acta Gastroenterol Latinoam 2020;50(3):271-278

Recibido: 28/02/2020 / Aceptado: 27//07/2020 / Publicado online: 28/09/2020

\section{Resumen}

El objetivo del estudio fue describir las caracteristicas clinicas y la proporción de las distintas etiologias de la cirrosis hepática (CH) diagnosticadas entre los años 2010 y 2017 y comparar con los resultados obtenidos durante el periodo 1995-2002. En el periodo 2010-2017 se diagnosticaron 260 pacientes con $\mathrm{CH}$. Las etiologías fueron: alcohol etílico en 94 pacientes $(36,1 \%)$, hepatitis crónica $C(H C V)$ en 87 (33,4\%), enfermedad del higado graso no alcohólico (EHG$N A)$ en 35 (13,4\%), colangitis biliar primaria (CBP) en 13 (5,0\%), hepatitis autoinmune (HAI) en 12 (4,6\%), hepatitis crónica $B$ en 7 (2,7\%), hemocromatosis hereditaria en $1(0,4 \%)$ y criptogénica en 11 (4,2\%). En comparación con el periodo de 1995-2002 que incluyó 252 pacientes, hubo una reducción significativa del número y porcentaje de la
$\mathrm{CH}$ por alcohol y criptogénica y un incremento de las asociadas a EHGNA y HAI. Comparativamente con el periodo de 1995-2002, se observó una mejor función hepática de la $\mathrm{CH}$ en general y en especial en las vinculadas al alcohol y a HCV. Sin embargo, en el periodo 2010-2017, las $\mathrm{CH}$ por alcohol comparadas con las asociadas a HCV e EHGNA presentaron un deterioro significativo mayor de la función hepática. Las principales etiologías de la $\mathrm{CH}$ son el alcoholy la HCV, seguidas por el EHGNA y las enfermedades hepáticas autoinmunes (CBP y HAI). Aunque en el periodo actual la $\mathrm{CH}$ se diagnostica en estadios más tempranos, la $\mathrm{CH}$ por alcohol presentó un bajo porcentaje de diagnósticos iniciales en la etapa asintomática.

Palabras claves. Cirrosis hepática, alcohol, hepatitis $C$, enfermedad del hígado graso no alcohólico.

\section{Abstract etiology of cirrhosis: epidemi- ological changes between the periods 1995-2002 and 2010-2017}

\section{Summary}

The aim of the study was to describe the clinical characteristics and the proportion of the different etiologies of liver cirrhosis (LC) diagnosed between 2010 and 2017 and compare with the period 1995-2002. In the 2010-2017 period, 260 patients with LC were diagnosed. The etiologies were: 
Alcohol 94 patients (36.1\%), chronic hepatitis C (HCV) 87 (33.4\%), non-alcoholic fatty liver disease (NAFLD) 35 (13.4\%), primary biliary cholangitis 13 (PBC) (5.0\%), autoimmune hepatitis (AIH) 12 (4.6\%), chronic hepatitis $B$ 7 (2.7\%), hereditary hemochromatosis 1 (0.4\%) and cryptogenic 11 (4.2\%). Compared with the 1995-2002 period that included 252 patients, there was a significant reduction in the number and percentage of $L C$ by alcohol and cryptogenic and an increase in those associated with NAFLD and AIH. In comparison with the period 1995-2002, a better hepatic function of LC was observed in general, and especially in those related to alcohol and HCV. However, in the 2010-2017 period, LC by alcohol compared with those associated with HCV and NAFLD showed a significantly greater deterioration in liver function. The main etiologies of $L C$ are alcohol and HCV followed by NAFLD and autoimmune liver diseases (PBC and AIH). Although in the current period $L C$ was diagnosed at earlier stages, LC by alcohol presented a low percentage of initial diagnoses in the asymptomatic stage.

Key words. Liver cirrhosis, alcohol, hepatitis $C$, non-alcoholic fatty liver disease.

\section{Abreviaturas}

CH: Cirrosis hepática.

$H B V$ : Hepatitis viral crónica por virus $B$.

$H C V$ : Hepatitis viral crónica por virus $C$ / Chronic Hepatitis $C$. EHGNA: Enfermedad del hígado graso no alcohólico.

LC: Liver cirrhosis.

NAFLD: Non-Alcoholic Fatty Liver Disease.

CBP: Colangitis biliar primaria.

HAI: Hepatitis autoinmune.

HH: Hemocromatosis hereditaria.

VEG: Várices esófago gástricas.

EDA: Endoscopía digestiva alta.

\section{Introducción}

La cirrosis hepática $(\mathrm{CH})$ constituye un importante problema de salud pública. ${ }^{1}$ Causó la pérdida de $31 \mathrm{mi}$ llones de años de vida ajustados por discapacidad (1,2\% de los globales) y el $2 \%$ de todas las muertes en el mundo en el año 2010. ${ }^{1,2}$ En Argentina se estima que en ese mismo año murieron por $\mathrm{CH} 6520$ personas, correspondiendo a 16 muertes por cada 100000 personas por año. ${ }^{3}$

Distintas enfermedades hepáticas crónicas como las provocadas por el alcohol etílico, las hepatitis virales crónicas por virus $\mathrm{B}(\mathrm{HBV})$ y virus $\mathrm{C}(\mathrm{HCV})$, las enferme- dad del hígado graso no alcohólico (EHGNA), la colangitis biliar primaria (CBP), la hepatitis autoinmune (HAI) y la hemocromatosis hereditaria $(\mathrm{HH})$ en su evolución pueden progresar a una $\mathrm{CH}$ sin dar síntomas. Una vez establecida la $\mathrm{CH}$, la probabilidad de la aparición de complicaciones como una hemorragia digestiva por várices esófago gástricas (VEG), ascitis, encefalopatía hepática, insuficiencia hepática y hepatocarcinoma se incrementa significativamente con una mortalidad al año que oscila entre $1 \%$ y $57 \%$, según el estadio de severidad. ${ }^{4} \mathrm{El}$ diagnóstico temprano de la $\mathrm{CH}$ constituye un importante objetivo en la práctica asistencial debido a que la mayoría de sus causas son tratables y, de esta manera, es posible evitar la progresión y aparición de complicaciones con la consiguiente mejoría en la sobrevida de los pacientes. ${ }^{5}$

Establecer la etiología de la enfermedad hepática crónica en cualquiera de sus diferentes estadios es el paso inicial en el manejo de estas entidades. La frecuencia de cada etiología variará de acuerdo a las características epidemiológicas de cada región, país, provincia o municipio. En Argentina, en los últimos años, han existido cambios en el nivel y patrones del consumo de alcohol, ${ }^{6}$ un incremento de la prevalencia del sobrepeso, obesidad y diabetes, ${ }^{7}$ una progresión a estadios avanzados de los portadores $\mathrm{HCV}$ sin diagnóstico, ${ }^{8}$ la introducción de nuevos fármacos antivirales de alta eficacia en $\mathrm{HBV}$ y $\mathrm{HCV}^{9,10}$ y una mayor incidencia y reconocimiento de las enfermedades hepáticas autoinmunes ${ }^{11}$ que influyen en la frecuencia observada de cada una de las etiologías de la $\mathrm{CH}$.

El surgimiento de métodos no invasivos para evaluar el estadio de fibrosis ha permitido la detección de los pacientes con fibrosis avanzada o $\mathrm{CH}$ en el período asintomático previo a la aparición de las complicaciones y el deterioro de la función hepática. ${ }^{12}$ Esta metodología implementada en los últimos años ha cambiado las características epidemiológicas de los pacientes en el momento inicial del diagnóstico, como consecuencia de su mayor aplicabilidad y aceptación debido a la ausencia de complicaciones, a diferencia de lo que ocurre con la biopsia hepática. ${ }^{13}$

No hay estudios recientes, en nuestro medio, dirigidos específicamente a evaluar en general la frecuencia y proporción de las distintas etiologías y si han existido cambios temporales que permitan a los integrantes del equipo de salud y a la comunidad en general establecer pautas para la prevención, la detección precoz y el tratamiento adecuado de la enfermedad hepática crónica. Tampoco hay estudios que indiquen si en la evaluación inicial han existido modificaciones en su severidad.

El objetivo del presente estudio fue describir las características clínicas y la proporción de las distintas etiologías 
de la CH diagnosticadas entre los años 2010 y 2017 y comparar con los casos entre los años 1995 y 2002 en el Servicio de Hepatología del Sanatorio Dr. Julio Méndez.

\section{Material y métodos}

Se incluyeron en forma consecutiva pacientes con $\mathrm{CH}$ cuyos diagnósticos fueron realizados o confirmados por primera vez en el Servicio de Hepatología en dos períodos de 8 años; el primer período abarca desde enero de 2010 a diciembre de 2017 y el segundo, como control histórico, desde enero de 1995 a diciembre de 2002. Este estudio observacional retrospectivo se ajusta a las normas éticas de la declaración de Helsinki de 2013 y fue aprobado por el comité de revisión institucional.

El diagnóstico de la $\mathrm{CH}$ se realizó mediante una evaluación basada en el examen clínico, los estudios de laboratorio, las imágenes hepáticas, la endoscopía digestiva alta (EDA), la biopsia hepática y/o la elastografía hepática. Las etiologías de la $\mathrm{CH}$ se determinaron de acuerdo a los siguientes criterios: a) Alcohol: ingesta semanal mayor a $140 \mathrm{~g}$ la mujer y $210 \mathrm{~g}$ el hombre al menos durante 5 años, sin la presencia de HBV, HCV, HAI o CBP; ${ }^{14}$ b) HBV o HCV: serología positiva para HBsAg y AntiHCV de $3^{\circ}$ generación respectivamente; ${ }^{9} 10$ c) HAI o CBP: existencia de dos de los tres criterios que definen a estas entidades (patrón bioquímico, autoanticuerpos e histología característica); ${ }^{15}{ }^{16}$ d) $\mathrm{HH}$ : presencia de sobrecarga de hierro humoral y hepática con estudio genético compatible; ${ }^{17}$ e) EHGNA: existencia de obesidad y los componentes del síndrome metabólico en ausencia de las causas mencionadas anteriormente; ${ }^{18}$ y f) Criptogénica: ausencia de todas las causas mencionadas y sin otro motivo evidente.

Se registraron las siguientes variables: la edad, el sexo, la causa de la $\mathrm{CH}$, el grado y el puntaje de Child-Pugh, ${ }^{19}$ la presencia de VEG, el método de diagnóstico y la etiología.

De acuerdo a los valores asignados por el Child-Pugh se clasificó a la $\mathrm{CH}$ en: Child-Pugh A o cirrosis compensada (puntaje 5 ó 6), B (7 a 9) y C (10 a 15). Las CH ChildPugh B y C se clasificaron como cirrosis descompensadas.

La presencia de VEG se determinó por el resultado de la EDA cuando hubiese sido realizada.

Los datos fueron anónimos y registrados en una planilla de Excel Microsoft 2010®.

Según el análisis estadístico, las variables numéricas fueron expresadas como media \pm desvío estándar o mediana e intervalo intercuartil y las categóricas en porcentajes. Para la comparación de las variables numéricas se utilizaron la prueba t de Student y U Mann-Withney, según correspondiera, y para comparar proporciones la prueba de Chi cuadrado. Un valor de $p<0,05$ se consideró significativo. El programa con el cual se realizó el análisis estadístico fue el SPSS Statistics versión 21.

\section{Resultados}

Se diagnosticó la $\mathrm{CH}$ a 260 pacientes entre 2010 y 2017 y a 252 personas entre 1995 y 2002. La descripción y comparación de las muestras se presenta en la tabla 1 . La edad fue significativamente menor en el período 20102017 y no hubo diferencias significativas en relación al sexo. En ambos períodos, el método de diagnóstico de la $\mathrm{CH}$ fue clínico en la mayoría de los casos y, en menor frecuencia, histológico y por elastografía hepática. En relación al período 1995-2002 se observó una utilización menor de la biopsia hepática y un uso mayor de la elastografía hepática no disponible en aquel período.

Las etiologías halladas en el período 2010-2017 fueron: alcohol en 94 pacientes (36,1\%; IC 95\%: 30,542,1), HCV en 87 (33,5\%; IC 95\%: 28,0-39,4), EHGNA en 35 (13,5\%; IC 95\%: 9,8-18,1), CBP en 13 (5,0\%; IC 95\%: 2,9-8,3), HAI en 12 (4,6\%; IC 95\%: 2,6-7,8), HBV en 7 (2,7\%; IC 95\%: 1,3-5,4), HH en $1(0,4 \%$; IC 95\%: 0,0-2,1) y criptogénica en $11(4,2 \%$; IC 95\%: 2,3-7,4).

En comparación con el período 1995-2002 no se observaron cambios significativos en el número total de $\mathrm{CH}$. En cambio, hubo una reducción significativa del número y porcentaje de pacientes con $\mathrm{CH}$ por alcohol y $\mathrm{CH}$ criptogénica. Existió un incremento significativo de las $\mathrm{CH}$ asociadas a EHGNA y a la HAI (Tabla 1).

En relación a la función hepática se observó que la $\mathrm{CH}$ era predominantemente compensada y en comparación con el período 1995-2002 hubo una proporción significativamente mayor de $\mathrm{CH}$ compensada con una mediana del puntaje de Child-Pugh inferior (Tabla 1).

En los que tuvieron EDA, 195 pacientes $(75 \%)$ en el período 2010-2017 comparados con los 241 (95,6\%) del período 1995-2002, se observó una frecuencia significativamente menor de VEG [113/195 (57,9\%) vs. 170/241 (70,5\%); $p=0,003$ ]. Los que presentaban $\mathrm{CH}$ compensada no mostraron diferencias llamativas [71/127 $(55,9 \%)$ vs. $72 / 116(62,0 \%) ; p=0,164]$, pero en los que tenían $\mathrm{CH}$ descompensada la disminución alcanzó una significancia estadística [42/68 (61,7\%) vs. 98/125 $(72,5 \%) ; p=0,006]$.

En relación a la $\mathrm{CH}$ por alcohol, se observaron diferencias considerables respecto a la menor proporción y al número de casos anuales, una mejor función hepática expresada por un menor puntaje de Child-Pugh y una menor presencia de VEG, en general, en $\mathrm{CH}$ compen- 
Tabla 1. Características de las poblaciones estudiadas en los periodos 2010-2017 y 1995-2002

\begin{tabular}{|c|c|c|c|}
\hline & Período 2010-2017 & Período 1995-2002 & $p$ \\
\hline Pacientes $n$ & 260 & 252 & \\
\hline Edad media $\pm \mathrm{DE}$ & $59,4 \pm 11,8$ & $62,9 \pm 11,3$ & 0,001 \\
\hline Sexo femenino/ masculino $n(\%)$ & $99(38,1) / 161(61,9)$ & $90(35,7) / 162(64,3)$ & 0,289 \\
\hline Diagnóstico clínico n (\%) & $216(83,1)$ & $214(84,9)$ & 0,284 \\
\hline Diagnóstico por biopsia n (\%) & $20(7,7)$ & $38(15,1)$ & 0,004 \\
\hline Diagnóstico por elastografía n (\%) & $24(9,2)$ & $0(0,0)$ & $<0,001$ \\
\hline Alcohol n (\%) & $94(36,1)$ & $130(51,6)$ & $<0,001$ \\
\hline HCV n (\%) & $87(33,5)$ & $76(30,1)$ & 0,211 \\
\hline EHGNA n (\%) & $35(13,5)$ & $0(0,0)$ & $<0,001$ \\
\hline HBV n (\%) & $7(2,7)$ & $10(4,0)$ & 0,210 \\
\hline CBP n (\%) & $13(5,0)$ & $10(4,0)$ & 0,286 \\
\hline HAI n (\%) & $12(4,6)$ & $4(1,6)$ & 0,043 \\
\hline $\mathrm{HH} n(\%)$ & $1(0,4)$ & $2(0,8)$ & 0,489 \\
\hline Criptogénica n (\%) & $11(4,2)$ & $20(7,9)$ & 0,039 \\
\hline Child-Pugh A n (\%) & $168(64,6)$ & $124(49,2)$ & 0,000 \\
\hline Child-Pugh B n (\%) & $64(24,6)$ & $72(28,6)$ & 0,156 \\
\hline Child-Pugh C n (\%) & $28(10,8)$ & $56(22,2)$ & $<0,001$ \\
\hline Puntaje Child-Pugh mediana (RIQ) & $5,0(5-7)$ & $7,0(5-9)$ & $<0,001$ \\
\hline Casos anuales mediana (RIQ) & $31,5(27-36)$ & $31,5(26-39)$ & 0,959 \\
\hline
\end{tabular}

DE: desvío estándar; HCV: hepatitis viral crónica por virus C; EHGNA: enfermedad del hígado graso no alcohólico; HBV: hepatitis viral crónica por virus B; CBP: colangitis biliar primaria; HAl: hepatitis autoinmune; HH: hemocromatosis hereditaria; RIQ: rango intercuartil; EDA: endoscopía digestiva alta; VEG: várices esófago gástricas; $\mathbf{C H}$ : cirrosis hepática.

sada y descompensada, en comparación con el período 1995-2002 (Tabla 2).

Con respecto a la $\mathrm{CH}$ por HCV (Tabla 3), hubo un número de casos anuales similar en relación al período 1995-2002, pero los pacientes presentaron una importante edad menor de diagnóstico de la $\mathrm{CH}$ y mayor proporción de $\mathrm{CH}$ compensada en relación al período 1995-2002. Los pacientes con CH por HCV no mostraron diferencias interesantes en la prevalencia de VEG en comparación con el período 1995-2002.
La $\mathrm{CH}$ por alcohol, en comparación con la asociada a HCV diagnosticada en el período 2010-2017 (Tabla 4), presentó un aumento significativo de la edad y del sexo masculino y un deterioro significativo de la función hepática evidenciada por el grado y puntaje de Child-Pugh. En el período 2010-2017 se realizó un bajo porcentaje de los diagnósticos iniciales de la $\mathrm{CH}$ por alcohol en el período asintomático, en comparación con la $\mathrm{CH}$ por $\mathrm{HCV}$. Lo mismo ocurrió al cotejar la $\mathrm{CH}$ por EHGNA (Tabla 5). 
Tabla 2. Función hepática y presencia de várices esófago gástricas en cirrosis por alcohol en los periodos 2010-2017 y 1995-2002

\begin{tabular}{lccc}
\hline & Período 2010-2017 & Período 1995-2002 & $p$ \\
\hline Pacientes $n$ & 94 & 130 & 0,672 \\
Edad media \pm DE & $62,1 \pm 10,3$ & $61,5 \pm 10,6$ & 0,398 \\
Sexo femenino/ masculino n (\%) & $14(14,9) / 80(85,1)$ & $25(19,2) / 105(80,8)$ & 0,058 \\
Diagnóstico clínico $n$ (\%) & $93(98,9)$ & $122(93,8)$ & 0,058 \\
Diagnóstico por biopsia n (\%) & $1(1,1)$ & $8(6,2)$ & 0,068 \\
Diagnóstico por elastografía n (\%) & $0(0,0)$ & $48(36,9)$ & 0,915 \\
Child-Pugh A $n$ (\%) & $44(46,8)$ & $42(32,3)$ & 0,038 \\
Child-Pugh B $n$ (\%) & $31(33,0)$ & $40(30,8)$ & 0,034 \\
Child-Pugh C $n$ (\%) & $19(20,2)$ & $7,5(5-10)$ & $<0,001$ \\
Puntaje Child-Pugh mediana (RIQ) & $7,0(5-9)$ & $103 / 126(81,7)$ & 0,003 \\
Con EDA y con VEG n/n (\%) & $40 / 69(57,9)$ & $40 / 47(85,1)$ & 0,006 \\
Compensada con EDA y VEG n/n (\%) & $18 / 31(58,1)$ & $63 / 79(79,7)$ & 0,045 \\
Descompensada con EDA y VEG n/n (\%) & $22 / 38(57,9)$ & $16,5(12-19)$ & \\
Casos anuales mediana (RIQ) & $11,0(9-13)$ & \\
\hline
\end{tabular}

DE: desvío estándar; RIQ: rango intercuartil; EDA: endoscopía digestiva alta; VEG: várices esófago gástricas.

Tabla 3. Función hepática y presencia de várices esófago gástricas en cirrosis por HCV en los períodos 2010-2017 y 1995-2002

\begin{tabular}{lccc}
\hline & Período 2010-2017 & Período 1995-2002 & $p$ \\
\hline Pacientes $n$ & 87 & 76 & $<0,001$ \\
Edad media \pm DE & $54,2 \pm 10,6$ & $63,3 \pm 12,4$ & 0,094 \\
Sexo femenino/ masculino n (\%) & $30(34,5) / 57(65,5)$ & $36(47,4) / 40(52,6)$ & 0,157 \\
Diagnóstico clínico n (\%) & $59(67,8)$ & $57(75,0)$ & 0,003 \\
Diagnóstico por biopsia n (\%) & $8(9,2)$ & $19(25,0)$ & $<0,001$ \\
Diagnóstico por elastografía n (\%) & $0(0,0)$ & 0,004 \\
Child-Pugh A n (\%) & $20(23,0)$ & $47(61,8)$ & 0,023 \\
Child-Pugh B n (\%) & $70(80,5)$ & $21(27,6)$ & 0,126 \\
Child-Pugh C n (\%) & $13(14,9)$ & $8(10,5)$ & 0,014 \\
Puntaje Child-Pugh mediana (RIQ) & $4(4,6)$ & $5,5(5-8)$ & 0,370 \\
Con EDA y con VEG n/n (\%) & $5,0(5-6)$ & $38 / 73(52,1)$ & 0,526 \\
Compensada con EDA y VEG n/n (\%) & $33 / 67(49,2)$ & $18 / 44(40,9)$ & 0,385 \\
Descompensada con EDA y VEG n/n (\%) & $26 / 55(47,3)$ & $20 / 29(69,0)$ & 0,455 \\
Casos anuales mediana (RIQ) & $7 / 12(58,3)$ & $9,5(6-13)$ & \\
\hline
\end{tabular}

DE: desvío estándar; RIQ: rango intercuartil; EDA: endoscopía digestiva alta; VEG: várices esófago gástricas. 
Tabla 4. Función hepática y presencia de várices esófago gástricas en cirrosis por alcohol y por HCV comparadas en el período 2010-2017

\begin{tabular}{|c|c|c|c|}
\hline & Cirrosis por alcohol & Cirrosis por HCV & $p$ \\
\hline Pacientes $n$ & 94 & 87 & \\
\hline Edad media $\pm \mathrm{DE}$ & $62,1 \pm 10,3$ & $54,2 \pm 10,6$ & $<0,001$ \\
\hline Sexo femenino/ masculino n (\%) & $14(14,9) / 80(85,1)$ & $30(34,5) / 57(65,5)$ & 0,002 \\
\hline Diagnóstico clínico n (\%) & $93(98,9)$ & $59(67,8)$ & $<0,001$ \\
\hline Diagnóstico por biopsia n (\%) & $1(1,1)$ & $8(9,2)$ & 0,014 \\
\hline Diagnóstico por elastografía n (\%) & $0(0,0)$ & $20(23,0)$ & $<0,001$ \\
\hline Child-Pugh A n (\%) & $44(46,8)$ & $70(80,5)$ & $<0,001$ \\
\hline Child-Pugh B n (\%) & $31(33,0)$ & $13(14,9)$ & 0,002 \\
\hline Child-Pugh C n (\%) & $19(20,2)$ & $4(4,6)$ & 0,001 \\
\hline Puntaje Child-Pugh mediana (RIQ) & $7,0(5-9)$ & $5,0(5-6)$ & $<0,001$ \\
\hline Con EDA y con VEG n/n (\%) & $40 / 69(57,9)$ & $33 / 67(49,2)$ & 0,155 \\
\hline Compensada con EDA y VEG n/n (\%) & $18 / 31(58,1)$ & $26 / 55(47,3)$ & 0,168 \\
\hline Descompensada con EDA y VEG n/n (\%) & $22 / 38(57,9)$ & $7 / 12(58,3)$ & 0,651 \\
\hline Casos anuales mediana (RIQ) & $11,0(9-13)$ & $10,0(10-13)$ & 0,402 \\
\hline
\end{tabular}

DE: desvío estándar; RIQ: rango intercuartil; EDA: endoscopía digestiva alta; VEG: várices esófago gástricas.

Tabla 5. Función hepática y presencia de várices esófago gástricas en cirrosis por alcohol y por EHGNA comparadas en el periodo 2010-2017

\begin{tabular}{lccr}
\hline & Cirrosis por alcohol & Cirrosis por EHGNA & $p$ \\
\hline Pacientes $n$ & 94 & 35 & 0,508 \\
Edad media \pm DE & $62,1 \pm 10,3$ & $62,7 \pm 9,3$ & $<0,001$ \\
Sexo femenino/ masculino n (\%) & $14(14,9) / 80(85,1)$ & $22(62,9) / 13(37,1)$ & 0,013 \\
Diagnóstico clínico n (\%) & $93(98,9)$ & $31(85,6)$ & 0,013 \\
Diagnóstico por biopsia $n$ (\%) & $1(1,1)$ & $4(11,4)$ & \\
Diagnóstico por elastografía n (\%) & $0(0,0)$ & $0(0,0)$ & 0,006 \\
Child-Pugh A n (\%) & $44(46,8)$ & $25(71,4)$ & 0,316 \\
Child-Pugh B n (\%) & $31(33,0)$ & $10(28,6)$ & 0,001 \\
Child-Pugh C $n$ (\%) & $19(20,2)$ & $0(0,0)$ & 0,001 \\
Puntaje Child-Pugh mediana (RIQ) & $7,0(5-9)$ & $5,0(5-7)$ & 0,255 \\
Con EDA y con VEG n/n (\%) & $40 / 69(57,9)$ & $17 / 26(65,4)$ & 0,360 \\
Compensada con EDA y VEG n/n (\%) & $18 / 31(58,1)$ & $12 / 19(63,2)$ & 0,083 \\
Descompensada con EDA y VEG n/n (\%) & $22 / 38(57,9)$ & $6 / 7(85,7)$ & 0,001 \\
Casos anuales mediana (RIQ) & $11,0(9-13)$ & $4,0(2-6)$ & \\
\hline EHGNA: enfermedad del hígado graso no alcohólico; DE: desvío estándar; RIQ: rango intercuartil; EDA: endoscopía digestiva alta;
\end{tabular}




\section{Discusión}

En la actualidad y en nuestra institución la $\mathrm{CH}$ es una causa importante de enfermedad hepática crónica que no ha variado su frecuencia desde el año 1995 cuando fue creado el servicio de Hepatología del Sanatorio Julio Méndez.

La metodología diagnóstica fue principalmente basada en el examen clínico y estudios complementarios de bajos costos y accesibles. Un avance significativo en el diagnóstico de la $\mathrm{CH}$ ha sido la introducción de la elastografía hepática, un método no invasivo, que valora a través del ultrasonido la rigidez hepática, un subrogante del grado de fibrosis hepática. Este método exento de complicaciones permite realizar el diagnóstico de la $\mathrm{CH}$ en un grupo adicional de pacientes donde su presencia aún no es evidente en la evaluación clínica. Este método, disponible en nuestra institución a partir del año 2014, permitió hacer el diagnóstico de la $\mathrm{CH}$ en un $9 \%$ de los casos y explicaría la menor utilización de biopsias hepáticas en la actualidad, especialmente en los pacientes con hepatitis crónica $\mathrm{C}$ donde el método ha mostrado una mejor validez diagnóstica. ${ }^{7}$

En nuestro estudio, a pesar de una reducción vinculada al consumo de alcohol significativa en el período 2010-2017, las principales etiologías de la $\mathrm{CH}$ fueron el alcohol y la HCV, seguidas en frecuencia por la asociada a EHGNA y a enfermedades autoinmunes hepáticas (CBP y HAI). Consideramos que esta reducción de la $\mathrm{CH}$ por alcohol se vincularía a dos motivos: a la derivación temprana de los pacientes a una consulta especializada por el consumo excesivo de alcohol antes del desarrollo de una enfermedad hepática avanzada con la aparición de complicaciones y al cambio del patrón del consumo de alcohol en Argentina que ha pasado de la ingesta excesiva regular y diaria a la episódica los fines de semana. ${ }^{20} \mathrm{El}$ consumo episódico excesivo o binge drinking está también vinculado a la enfermedad hepática crónica avanza$\mathrm{da}$, pero en menor frecuencia que con el consumo regular excesivo. ${ }^{21}$

$\mathrm{El}$ incremento de los diagnósticos de la $\mathrm{CH}$ por EHGNA podría ser consecuencia del aumento de la prevalencia del sobrepeso y la obesidad en la población, especialmente en la Ciudad Autónoma de Buenos Aires y en la provincia de Buenos Aires de donde provienen la mayoría de los pacientes incluidos en nuestro estudio, ${ }^{7} \mathrm{y}$ de la menor disponibilidad de información para aplicar los criterios de diagnóstico de la $\mathrm{CH}$ por EHGNA en el período $1995-2002 .{ }^{18}$ Esto último podría explicar también la disminución de los casos de la $\mathrm{CH}$ criptogénica en relación al período 1995-2002 donde algunas de ellas podrían corresponder a EHGNA. De todos modos, esto por sí solo no explicaría el aumento de la CH por EHGNA observado en el período 2010-2017.

El hallazgo de una mejor función hepática y de una menor prevalencia de VEG en los pacientes con $\mathrm{CH}$ comparado con el período 1995-2002, indicaría que el diagnóstico de $\mathrm{CH}$ fue realizado más frecuentemente en estadios tempranos. Pero a pesar de esta mejora en la detección temprana de la $\mathrm{CH}$, cuando comparamos las etiologías HCV y EHGNA en el período 2010-2017 con las provocadas por alcohol, hallamos una detección significativamente menor de la $\mathrm{CH}$ compensada, inferior al 50\%. Este descubrimiento coincide con los de otros estudios donde la $\mathrm{CH}$ por alcohol es hallada en estadios más avanzados que la vinculada a $\mathrm{HCV}^{22}$ e EHGNA. ${ }^{23}$ Esta observación hace énfasis en la necesidad de detectar sistemáticamente la presencia del consumo excesivo de alcohol en la atención primaria y realizar una adecuada evaluación de las consecuencias psico-socio-físicas, que incluya la evaluación de la existencia de la enfermedad hepática en el período asintomático. ${ }^{20}$

Hallar la $\mathrm{CH}$ con una mejor función hepática y con una menor prevalencia de la hipertensión portal es necesario para que las terapéuticas etiológicas, como la abstinencia de alcohol, la curación de la hepatitis $\mathrm{C}$, la supresión de la replicación del virus $\mathrm{B}$, el descenso de peso y la supresión de los fenómenos autoinmunes, tengan una mayor eficacia en evitar la progresión de la $\mathrm{CH}$ a estadios avanzados. ${ }^{10} \mathrm{La}$ adecuada evaluación clínica, los parámetros bioquímicos, las imágenes y los métodos no invasivos para determinar la severidad de la fibrosis permitieron en nuestra población actual el diagnóstico de la $\mathrm{CH}$ en más del $90 \%$ de los casos, de los cuales dos tercios tenían una función hepática conservada sin complicaciones.

La limitación más importante del estudio es la que corresponde a los estudios retrospectivos, en especial con el registro realizado entre 1995 y 2002. El registro realizado en este período tuvo un número limitado de variables que no nos permitieron evaluar la coexistencia de factores etiológicos asociados como consumo excesivo de alcohol en la $\mathrm{CH}$ por $\mathrm{HCV}$ o los factores asociados a EHGNA en pacientes con $\mathrm{CH}$ por $\mathrm{HCV}$, alcohol o criptogénica.

En conclusión, en nuestro estudio las principales etiologías de la $\mathrm{CH}$ son las asociadas al consumo de alcohol y la HCV, seguidas por las provocadas por el EHGNA y las enfermedades autoinmunes hepáticas. Destacamos el incremento de la $\mathrm{CH}$ por EHGNA posiblemente asociado al aumento de la prevalencia del sobrepeso y la obesidad en la población general de la Ciudad Autónoma de Buenos Aires y del conurbano bonaerense. Otro hallazgo de nuestro estudio fue la presencia de una mejor función hepática y una menor prevalencia de la hipertensión por- 
tal, lo cual indicaría un diagnóstico inicial en un estadio más temprano de la enfermedad hepática avanzada, cuando las medidas de tratamiento etiológico tienen mayor eficacia en la prevención de las complicaciones, mejoría del pronóstico y disminución de la mortalidad. Es deseable que esta tendencia a realizar un diagnóstico precoz de la enfermedad hepática crónica asociada a los factores etiológicos se incremente a fin de reducir en un futuro la frecuencia de la $\mathrm{CH}$ en la población, especialmente en la vinculada al consumo excesivo de alcohol.

\section{Conflicto de intereses. No hay conflicto de intereses.}

\section{Sostén financiero. Este documento no recibió ningún tipo de financiación o patrocinio externo.}

\section{Referencias}

1. Murray CJL, Vos T, Lozano R, Naghavi M, Flaxman AD, Michaud C, et al. Disability-adjusted life years (DALYs) for $291 \mathrm{di}$ seases and injuries in 21 regions, 1990-2010: a systematic analysis for the Global Burden of Disease Study 2010. The Lancet 2012; 380: P2197-2223.

2. Lozano R, Naghavi M, Foreman K, Lim S, Shibuya K, Aboyans $\mathrm{V}$, et al. Global and regional mortality from 235 causes of death for 20 age groups in 1990 and 2010: a systematic analysis for the Global Burden of Disease Study 2010. The Lancet 2012; 380: P2095-2128.

3. Mokdad AA, Lopez AD, Shahraz S, Lozano R, Mokdad AH, Stanaway J, Murray CJL, Naghavi M. Liver cirrhosis mortality in 187 countries between 1980 and 2010: a systematic analysis. BMC Med 2014; 12: 145.

4. D’Amico G, Garcia-Tsao G, Pagliaro L. Natural history and prognostic indicators of survival in cirrhosis: a systematic review of 118 studies. J Hepatol 2006; 44(1): 217-231.

5. Ge PS, Runyon BA. Treatment of Patients with Cirrhosis. N Engl J Med 2016; 375 (8): 767-777.

6. Informe de la Secretaría de Políticas Integrales sobre Drogas de la Nación Argentina (SEDRONAR). Tabaco - Alcohol. Intensidad del consumo. Estudio nacional en población de 12 a 65 años sobre consumo de sustancias psicoactivas. Argentina, 2017. Disponible en: http//www.observatorio.gov.ar/media/k2/attachments/InformeZconsumoZdeZTabaco_1.pdf 2017:1-42.

7. $3^{\circ}$ Encuesta Nacional de Factores de Riesgo para Enfermedades No Transmisibles. Presentación de los principales resultados. Ministerio de Salud de la Nación, Instituto Nacional de Estadísticas y Censos y las Direcciones Provinciales de Estadísticas 2013. Disponible en: http//msal.gob.ar/images/stories/publicaciones/pdf/11092014tercer-encuentro-nacional-factores-riesgo.pdf 2013.

8. Sibley A, Han KH, Abourached A, Lesmana LA, Makara M, Jafri $\mathrm{W}$, et al. The present and future disease burden of hepatitis $\mathrm{C}$ virus infections with today's treatment paradigm - volume 3 . J Viral Hepat 2015; 22: 21-41.
9. European Association for the Study of the Liver. EASL 2017 Clinical Practice Guidelines on the management of hepatitis B virus infection. J Hepatol 2017; 67 (2): 370-398. Disponible en: https://easl.eu/wp-content/uploads/2018/10/HepB-Englishreport.pdf.

10. European Association for the Study of the Liver. EASL Recommendations on Treatment of Hepatitis C 2018. J Hepatol 2018; 69 (2): 461-511.

11. Grønbæk L, Vilstrup H, Jepsen P. Autoimmune hepatitis in Denmark: Incidence, prevalence, prognosis, and causes of death. A nationwide registry-based cohort study. J Hepatol 2014; 60 (3): 612-617.

12. Berzigotti A, Ashkenazi E, Reverter E, Abraldes JG, Bosch J. Noninvasive diagnostic and prognostic evaluation of liver cirrhosis and portal hypertension. Dis Markers 2011; 31 (3): 129-138.

13. European Association for Study of Liver, Asociación Latinoamericana para el Estudio del Hígado. EASL-ALEH Clinical Practice Guidelines: Non-invasive tests for evaluation of liver disease severity and prognosis. J Hepatol 2015; 63 (1): 237-264.

14. Becker U, Deis A, Sørensen TI, Grønbaek M, Borch-Johnsen K, Müller CF, Schnohr P, Jensen G. Prediction of risk of liver disease by alcohol intake, sex, and age: a prospective population study. Hepatology 1996; 23 (5): 1025-1029.

15. European Association for the Study of the Liver. EASL Clinical Practice Guidelines: Autoimmune hepatitis. J Hepatol 2015; 63 (4): 971-1004.

16. European Association for the Study of the Liver. EASL Clinical Practice Guidelines: The diagnosis and management of patients with primary biliary cholangitis. J Hepatol 2017; 67 (1): 145-172.

17. European Association for the Study of the Liver. EASL clinical practice guidelines for HFE hemochromatosis. J Hepatol 2010; 53 (1): 3-22.

18. European Association for the Study of the Liver, European Association for the Study of Diabetes, European Association for the Study of Obesity. EASL-EASD-EASO Clinical Practice Guidelines for the management of non-alcoholic fatty liver disease. J Hepatol 2016; 64 (6): 1388-1402.

19. Pugh RN, Murray-Lyon IM, Dawson JL, Pietroni MC, Williams R. Transection of the oesophagus for bleeding oesophageal varices. Br J Surg 1973; 60 (8): 646-649.

20. Asociación Argentina para el Estudio de las Enfermedades del Hígado (AAEEH). Guías de Manejo de las Enfermedades Hepáticas Asociadas al Consumo de Alcohol 2018. Disponible en: http:// www.aaeeh.org.ar/es/attachment/show/46

21. Åberg F, Helenius-Hietala J, Puukka P, Jula A. Binge drinking and the risk of liver events: A population-based cohort study. Liver Int 2017; 37 (9): 1373-1381.

22. Shah ND, Ventura-Cots M, Abraldes JG, Alboraie M, Alfadhli A, Argemi J, et al. Alcohol-Related Liver Disease Is Rarely Detected at Early Stages Compared With Liver Diseases of Other Etiologies Worldwide. Clin Gastroenterol Hepatol 2019; 17 (11): 2320-2329.

23. Shoreibah M, Raff E, Bloomer J, Kakati D, Rasheed K, Kuo YF, Singal AK. Alcoholic liver disease presents at advanced stage and progresses faster compared to non-alcoholic fatty liver disease. Ann Hepatol 2016; 15 (2): 183-189. 